\title{
The Effect of Three Different Suture Anchors for Rotator Cuff Repair on Primary Cultures of Human Bone Marrow Mesenchymal Stem Cells
}

\author{
Gabriele Thiébat ${ }^{1}$ Paolo Capitani ${ }^{1}$ Laura de Girolamo ${ }^{2}$ Carlotta Perucca Orfei $^{2}$ \\ Francesca Facchini ${ }^{1}$ Herbert Schoenhuber ${ }^{1}$ Marco Viganò ${ }^{2}$
}

${ }^{1}$ Sport Traumatology Centre, Istituto Ortopedico Galeazzi, Milano, Italy

${ }^{2}$ Orthopaedic Biotechnology Laboratory, Istituto Ortopedico Galeazzi, Milano, Italy

Address for correspondence Paolo Capitani, MD, Sport Traumatology Centre, Istituto Ortopedico Galeazzi, via Riccardo Galeazzi 4, 20161 Milano, Italy (e-mail: paolocapitani.dr@gmail.com).

Joints 2018;6:100-103.

\begin{abstract}
Purpose The purpose of this study is to investigate the in vitro biocompatibility of three different suture anchors (all-suture anchor, metal anchor, and polyetheretherketone anchor), commonly used for the rotator cuff repair.

Methods To assess the biocompatibility of the anchors, the possible cytotoxicity and the immunogenicity of the devices were assessed by cell viability assay and cell count on cultures of bone marrow stem cells (BMSCs) and peripheral blood leucocytes (PBLs), respectively. The possible inhibitory effect of the devices on BMSCs osteogenic potential was evaluated by alkaline phosphatase activity and matrix deposition assay. Results The viability of BMSCs was slightly reduced when cultured in the presence of the devices $(-24 \pm 3 \%)$. Nevertheless, they were able to differentiate toward the

Keywords

- rotator cuff

- suture anchors

- bone marrow stem cells

- biocompatibility

- culture osteogenic lineage in all culture conditions. The proliferation of PBLs and the production of interleukin- 2 were not enhanced by the presence of any device.

Conclusion The analyzed devices did not significantly affect the normal cells functions when directly cultured with human primary BMSCs or PBLs, in terms of osteogenic differentiation and inflammatory reaction.

Clinical Relevance A deeper knowledge of the biological reactions to different devices used in rotator cuff surgeries would improve the clinical outcome of these procedures.
\end{abstract}

\section{Introduction}

The deeper knowledge achieved in the context of rotator cuff injuries and repair, together with the progressive optimization of the arthroscopic techniques, has made the arthroscopic rotator cuff repair a common and effective surgical procedure. Indeed, evidences of satisfactory outcomes of procedure are well reported in the literature, ${ }^{1}$ albeit a high recurrence rate is still described. ${ }^{2}$

Several factors, such as surgical technique, tissue quality, comorbidities and type of devices, have been reported to affect the outcome of arthroscopic rotator cuff repair. ${ }^{3}$ In particular, a growing interest has been recently focused on

received

September 5, 2017

accepted

Dol https://doi.org/

10.1055/s-0038-1660789.

ISSN 2282-4324. the biomechanical properties of different types of suture anchors used for rotator cuff repair concerning their stress resistance and suture configurations. ${ }^{4,5}$

The different types of anchors have specific advantages and disadvantages, resulting from their characteristics. The first anchors used in arthroscopic rotator cuff repair were metallic. They gave an excellent fixation but failure of the suture or migration of the anchor could lead to cartilage damage. ${ }^{6}$ Subsequently, biodegradable anchors were introduced. ${ }^{7}$ The first generation of biodegradable anchors had the advantage of combining the resistance to pullout of the metal anchors with a reduced signal alteration at the magnetic resonance imaging (MRI). However, complications were reported with the use of 
this type of suture anchors, such as suture failure due to early degradation, reactive synovitis, cyst formation, and osteolysis. ${ }^{8-11}$ Moreover, standard radiographs do not allow for the visualization of a mobilized biodegradable anchor, and MRI would be needed. ${ }^{12}$

The development of these devices continued to move forward and new anchors were recently introduced by using new materials. Nevertheless, little consideration has been given to the possible interaction between these fixation devices and the biological environment. Indeed, a low biocompatibility could represent a trigger for inflammation or bone resorption, possibly causing failure of the procedure.

The purpose of this study was to verify the in vitro biocompatibility of three different types (all-suture anchor, metal anchor, and polyetheretherketone [PEEK] anchor). The hypothesis of the study was that suture anchors can differ according to the material in terms of cytotoxicity, immunogenicity, and inhibitory action on osteogenic differentiation.

\section{Methods}

\section{Bone Marrow Stem Cells Isolation and Culture}

Human bone marrow stem cells (BMSCs) were collected from waste surgical samples at our institute under written consent of the patients (M-SPER-014.ver7 for the use of surgical waste). Cells were obtained from the femoral canal of one donor after hip surgical replacement. The aspirate was washed with phosphate-buffered saline (PBS) and centrifuged at $623 \mathrm{~g}$ for 10 minutes. The pellet containing the mononuclear cells fraction was resuspended in complete medium (CM), composed of Dulbecco's Modified Eagle Medium (DMEM) High Glucose (Sigma-Aldrich, St. Louis, Missouri, United States), 10\% fetal bovine serum (FBS, Sigma-Aldrich), $50 \mathrm{U} / \mathrm{mL}$ penicillin, 50 $\mathrm{mg} / \mathrm{mL}$ streptomycin, $2 \mathrm{mM}$ L-glutamine (Life Technologies, Carlsbad, California, United States) and plated in culture flasks at a density of $5 \times 10^{3}$ cells $/ \mathrm{cm}^{2}$. Cells were maintained in culture and expanded until passage 4 .

BMSCs were incubated with three different types of suture anchors: an all-suture anchor (JuggerKnot; Biomet, Warsaw, Indiana, United States), a metal anchor (ThRevo-FT; ConMed, Largo, Florida, United States), and a PEEK anchor (Cross-FT; ConMed). BMSC cultures without the devices were used as control samples. Cells were plated onto 12well plates at a seeding density of 10,000 cells $/ \mathrm{cm}^{2}$. Culture medium was the same for all specimens: a complete culture medium consisting of DMEM (Sigma-Aldrich), 10\% FBS (GE Healthcare, Chicago, Illinois, United States), and 1\% penicillin-streptomycin-glutamine (Life Technologies).

\section{Cell Viability}

Cell viability was assessed after 7 days of culture, by MTT colorimetric assay (3-(4,5-dimethylthiazol-2-yl)-2,5-diphenyltetrazolium bromide; Sigma-Aldrich). According to the protocol, specimens were incubated with MTT solution at a concentration of $0.5 \mathrm{mg} / \mathrm{mL}$ for 4 hours at a temperature of $37^{\circ} \mathrm{C}$. Following solubilization with $100 \%$ dimethyl sulfoxide (Sigma-Aldrich), absorbance was measured by spectrophotometry at $570 \mathrm{~nm}$ wavelength.

\section{Osteogenic Differentiation}

At passage 4, BMSCs were seeded at the density of $10^{4}$ cells $/ \mathrm{cm}^{2}$ in a 12-well plate. Osteogenic stimulus was provided by culturing cells in CM supplemented with dexamethasone (10 nM), glycerol 2-phosphate (10 mM), L-ascorbic acid 2-phosphate $(150 \mu \mathrm{M})$, and cholecalciferol $(10 \mathrm{nM})$. Cultures with each device were performed in duplicates and BMSCs without devices were considered as controls. Alkaline phosphatase (ALP) activity was assessed after 14 days of culture, by enzymatic assay. Briefly, specimens were lysed in $0.1 \%$ Triton X-100 and incubated at $37^{\circ}$ $\mathrm{C}$ with $1 \mathrm{mM}$ para-nitrophenyl phosphate in phosphatase buffer $\left(100 \mathrm{mM}\right.$ diethanolamine and $0.5 \mathrm{mM} \mathrm{MgCl}_{2}$ ). The amount of chromogenic para-nitrophenol (pNP) product was assessed by spectrophotometer. Readings were taken at $405 \mathrm{~nm}$ wavelength, and ALP activity was normalized against the total protein content determined by bicinchoninic acid (BCA) protein assay (BCA Protein Assay Kit; Thermo Fisher Scientific, Waltham, Massachusetts, United States).

Calcified matrix deposition was assessed after 21 days of culture, by alizarin red staining (ARS). Cells were stained with $40 \mathrm{mM}$ Alizarin Red S for 15 minutes. Calcified matrixbound dye was extracted by incubation with cetylpyridinium chloride $(\mathrm{CPC})$ in phosphate buffer $(0.1 \mathrm{M})$ to quantify the amount of calcified matrix. Spectrophotometer readings were taken at $550 \mathrm{~nm}$ wavelength.

\section{Peripheral Blood Leucocytes Isolation and Culture}

Human peripheral blood leucocytes (PBLs) were isolated from $10 \mathrm{~mL}$ of peripheral blood from one healthy donor, under informed consent. Briefly, blood was diluted 1:1 with PBS layered over $10 \mathrm{~mL}$ Ficoll Isopaque (GE Healthcare) in a $50-\mathrm{mL}$ tube and centrifuged for 40 minutes at $400 \mathrm{~g}$. After centrifugation, the nucleated cells at the interface between Ficoll and supernatant were collected, washed two times with PBS, counted, and then maintained in RPMI medium with $10 \%$ FBS. Cells were then cultured in the presence or absence of the three different devices for 5 days.

\section{Data Analysis}

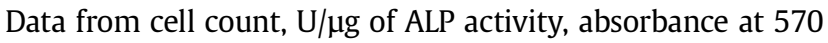
and $550 \mathrm{~nm}$ for cell viability and ARS staining, respectively, were expressed as means \pm standard deviation. Statistical analyses were performed with a statistical software (GraphPad Prism v5.00; GraphPad Software, La Jolla, California, United States) using the analysis of variance one-way test with Bonferroni's post hoc test for the evaluation of differences between single datasets. Level of significance was set at $p<0.05$.

\section{Results}

\section{Cell Viability}

Cell viability of BMSCs, incubated or not with the medical devices, was assessed after 7 days of culture by MTT assay. If compared with the control samples (without devices), significantly lower viability was observed for BMSCs incubated with ThRevo-FT ( $-22 \% ; p<0.05)$ and Cross-FT $(-28 \% ; p<0.05)$. However, no differences were observed among cultures incubated with the devices (-Fig. 1). 


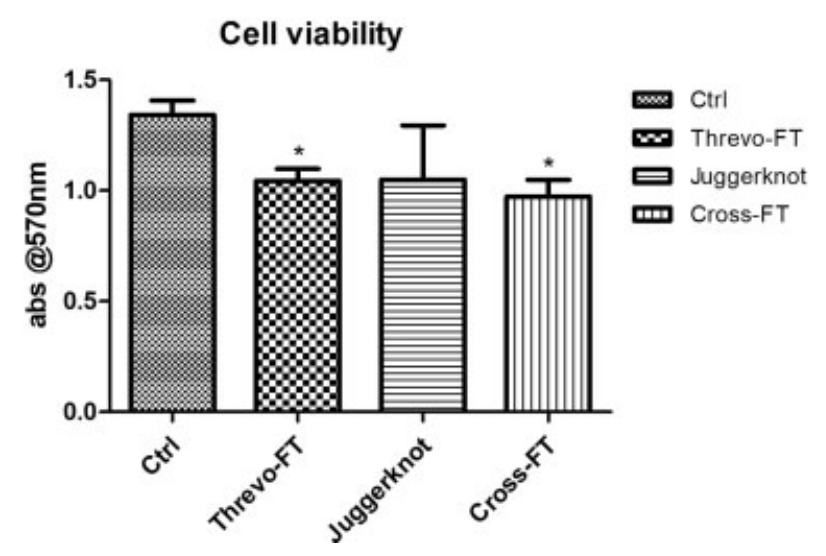

Fig. 1 Viability assessment of BMSCs after 7 days of culture with or without the suture anchors (data are expressed as means $\pm S D$ ). Control: ${ }^{*} p<0.05$. BMSCs, bone marrow stem cells; SD, standard deviation.

\section{Osteogenic Differentiation}

The ALP activity levels of osteo-differentiated BMSCs, both in the presence or absence of the devices, were higher than the undifferentiated ones. In particular, significantly higher mean values were observed for BMSCs alone $(+83 \%$; $p<0.01)$ and for BMSCs incubated with the ThRevo-FT anchors ( $+39 \% ; p<0.05)$. A nonsignificant increase of ALP activity was observed in samples in contact with JuggerKnot with respect to the control maintained in noninductive medium $(p=0.069)$. However, osteo-differentiated BMSCs cultured in the presence of the three different devices showed lower levels of ALP activity in comparison with osteo-differentiated BMSCs alone (-Fig. 2).

As expected, the deposition of calcified matrix of differentiated BMSCs was higher than one of the undifferentiated cells $(+1,551 \% ; p<0.001)$. Similarly, BMSCs cultured with the devices produced higher levels of calcified matrix in comparison with control samples, even if only tendency were found in the statistical analysis, due to high variability in the samples. In

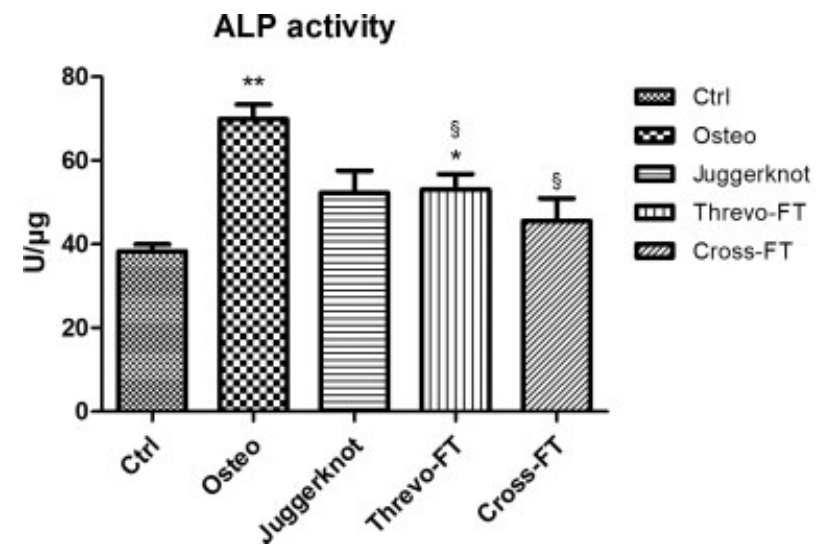

Fig. 2 ALP activity levels of osteo-differentiated BMSCs after 14 days of culture, in the presence or absence of the suture anchors. Values are normalized against the protein content of each sample (data are expressed as the mean of two replicates \pm SD). Control: ${ }^{*} p<0.05$; $^{* *} p<0.01$; osteodifferentiated BMSCs: ${ }^{\S} p<0.05$. ALP, alkaline phosphatase; BMSCs, bone marrow stem cells; SD, standard deviation.

\section{Calcified matrix deposition}

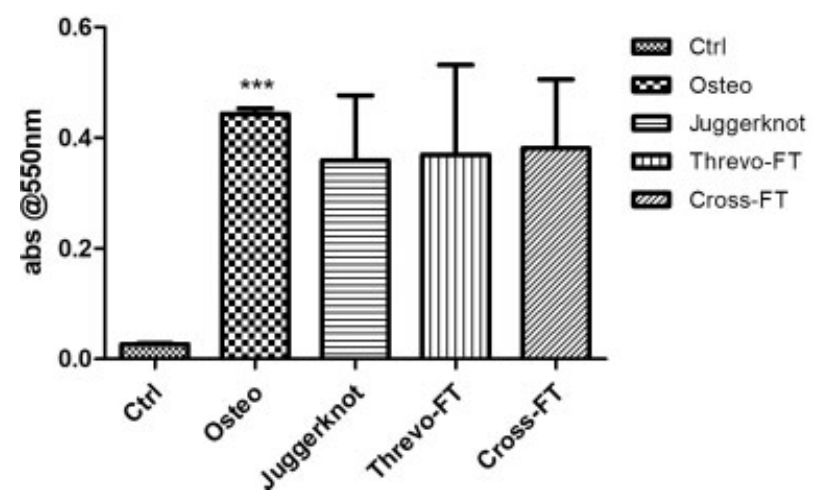

Fig. 3 Quantification of calcified matrix deposition by Alizarin red S staining and extraction, of osteo-differentiated BMSCs after 21 days of culture, in the presence or absence of the devices (data are expressed as the mean of two replicates \pm SD). Control: ${ }^{* * *} p<0.001$. BMSCs, bone marrow stem cells; SD, standard deviation.

particular, increases of $1,238 \%(p=0.057), 1,277 \%(p=0.096)$, and $+1,320 \%(p=0.056)$ were observed for JuggerKnot, ThRevo-FT, and Cross-FT, respectively (-Fig. 3).

\section{Immune Response Activation}

After 5 days of culture, cells were quantified by the Trypan blue staining method. The PBLs counts in the samples incubated with the devices were similar to those from the control group; hence, PBLs proliferation was not increased by the presence of the devices. On the contrary, slight decreases in the number of PBLs were detected in all the samples cultured in the presence of the devices ( - Fig. 4). Interleukin2 content was determined by enzyme-linked immunosorbent assay after 5 days in culture and it resulted undetectable for all specimens.

\section{Discussion}

Rotator cuff repair aims to restore the bone-tendon contact interface. Open and the arthroscopic transosseous repair technique restore the bone-tendon interface without the use of implant devices, other than the suture thread. ${ }^{13}$ However, standard arthroscopic rotator cuff repair took

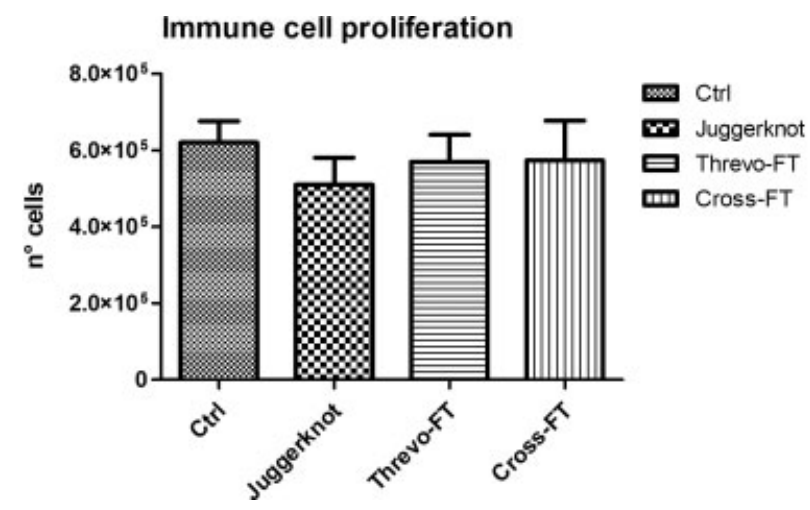

Fig. 4 Peripheral blood leucocytes count from each culture condition (data are expressed as the mean of two replicates \pm standard deviation). 
advantage of implantable devices, the suture anchors. These devices are within the bone-tendon interface and they should not hinder tendon-to-bone healing process. Osteolysis and cysts, described among the complications in the use of these devices, were supposed to have not only mechanical causes but also local biological causes in the interaction of the anchors with bone and soft tissues. ${ }^{7,14}$

Some previous studies focused on the different effects of these devices on the surrounding cellular and tissue environment. Unfortunately, several variables are involved in the modulation of healing processes and cellular signals, related to the implants and to the patients as well. Zhang et $\mathrm{al}^{15}$ reported that age and sex affect the ability of osteoblasts to produce bone. Furthermore, injured tendons were found to have different collagen-type composition compared with noninjured ones. ${ }^{16}$

Numerous modifications of the devices concerning material composition and coating have been proposed to improve their osseointegration and tissue healing. A different bone response, both in formation and in cells modulation, was found in vitro according to chemical processing of the devices. ${ }^{17}$

Mazzocca et $\mathrm{al}^{18}$ reported that a collagen-coated suture stimulates primary human osteoblasts and tenocytes to proliferate, to adhere, and to differentiate, compared with a noncoated suture. This was the first study that focused on the biocompatibility of suture anchors made of different materials on the same cellular environment in vitro.

In this study, BMSCs cultured in the presence of all-suture, metal, and PEEK anchors maintained their viability. Although cell viability resulted slightly decreased in all the samples cultured in the presence of the devices, if compared with the control, such reduction may have been provoked by the culture method. In fact, the direct contact of the devices with the cells may have hampered the normal cell growth.

The presence of the devices in direct culture with BMSCs did not prevent their differentiation ability toward the osteogenic lineage. Indeed, when maintained in osteogenic inductive medium, all the samples were able to deposit higher amount of calcified matrix with respect to the undifferentiated control. Similarly, ALP activity resulted higher in all samples, compared with control cells in noninductive medium. Despite a comparison with control cells in osteogenic medium showed a reduction in these parameters, the entity of such a decrease is insubstantial because all the samples resulted positive to calcified matrix deposition and ALP activity increased with respect to undifferentiated controls.

None of the investigated devices was found to elicit an inflammatory response when incubated with PBLs. The absence of PBL activation was confirmed by the lack of cell proliferation and IL-2 production in all the analyzed samples, supporting the nonimmunogenicity of the devices.

In conclusion, no relevant interference with normal cell functions was detected when BMSCs or PBLs were cultured in direct contact with all-suture, metal, and PEEK suture anchors. Further in vitro studies are needed to better evaluate the local effect of suture anchors and the biological pathways to reduce complications and improve tendon healing.

\section{Conflict of Interest}

Dr. de Girolamo reports personal fees from Lipogemsand Geistlichand grants from IGEA, outside the submitted work.

\section{References}

1 DeHaan AM, Axelrad TW, Kaye E, Silvestri L, Puskas B, Foster TE. Does double-row rotator cuff repair improve functional outcome of patients compared with single-row technique? A systematic review. Am J Sports Med 2012;40(05):1176-1185

2 Randelli P, Randelli F, Compagnoni R, et al. Revision reverse shoulder arthroplasty in failed shoulder arthroplasties for rotator cuff deficiency. Joints 2015;3(01):31-37

3 Saccomanno MF, Sircana G, Cazzato G, Donati F, Randelli P, Milano G. Prognostic factors influencing the outcome of rotator cuff repair: a systematic review. Knee Surg Sports Traumatol Arthrosc 2016;24(12):3809-3819

4 Nagra NSN, Zargar N, Smith RDJ, Carr AJ. Mechanical properties of all-suture anchors for rotator cuff repair. Bone Joint Res 2017;6 (02):82-89

5 Esquivel AO, Duncan DD, Dobrasevic N, Marsh SM, Lemos SE. Load to failure and stiffness: anchor placement and suture pattern effects on load to failure in rotator cuff repairs. Orthop J Sports Med 2015;3(04):2325967115579052

6 Goeminne S, Debeer P. Delayed migration of a metal suture anchor into the glenohumeral joint. Acta Orthop Belg 2010;76(06):834-837

7 Dhawan A, Ghodadra N, Karas V, Salata MJ, Cole BJ. Complications of bioabsorbable suture anchors in the shoulder. Am J Sports Med 2012;40(06):1424-1430

8 Kelly JD II. Disintegration of an absorbable rotator cuff anchor six weeks after implantation. Arthroscopy 2005;21(04):495-497

9 Park AY, Hatch JD. Proximal humerus osteolysis after revision rotator cuff repair with bioabsorbable suture anchors. Am J Orthop 2011;40(03):139-141

$10 \mathrm{Kim} \mathrm{SH}$, Oh JH, Lee OS, Lee HR, Hargens AR. Postoperative imaging of bioabsorbable anchors in rotator cuff repair. Am J Sports Med 2014;42(03):552-557

11 McCarty LP III, Buss DD, Datta MW, Freehill MQ Giveans MR. Complications observed following labral or rotator cuff repair with use of poly-L-lactic acid implants. J Bone Joint Surg Am 2013; 95(06):507-511

12 Medina G, Garofo G, D'Elia CO, Bitar AC, Castropil W, Schor B. Bioabsorbable suture anchor migration to the acromioclavicular joint: how far can these implants go? Case Rep Orthop 2014;2014:834896

13 Randelli P, Stoppani CA, Zaolino C, Menon A, Randelli F, Cabitza P. Advantages of arthroscopic rotator cuff repair with a transosseous duture technique: a prospective randomized controlled trial. Am J Sports Med 2017;45(09):2000-2009

14 Luyckx T, Debeer P. Management of full thickness rotator cuff tears. A survey amongst members of the Flemish Elbow and Shoulder Surgeons Society (FLESSS). Acta Orthop Belg 2010;76(01):14-21

15 Zhang H, Lewis CG, Aronow MS, Gronowicz GA. The effects of patient age on human osteoblasts' response to Ti-6Al-4V implants in vitro. J Orthop Res 2004;22(01):30-38

16 Maffulli N, Ewen SW, Waterston SW, Reaper J, Barrass V. Tenocytes from ruptured and tendinopathic Achilles tendons produce greater quantities of type III collagen than tenocytes from normal achilles tendons. An in vitro model of human tendon healing. Am J Sports Med 2000;28(04):499-505

17 Zreiqat H, Akin FA, Howlett CR, et al. Differentiation of human bone-derived cells grown on GRGDSP-peptide bound titanium surfaces. J Biomed Mater Res A 2003;64(01):105-113

18 Mazzocca AD, McCarthy MB, Arciero C, et al. Tendon and bone responses to a collagen-coated suture material. J Shoulder Elbow Surg 2007;16(5, Suppl):S222-S230 